\title{
Voltammogram "Landscapes" Aid Detection and Identification of In Vivo Electrochemical Signals
}

\author{
Paul E. M. Phillips and Jonathan A. Stamford* \\ Neurotransmission Laboratory, Academic Department of Anaesthesia and Intensive Care, St Bartholomew's and the Royal London School of \\ Medicine and Dentistry, Alexandra Wing, Royal London Hospital, Whitechapel, London E1 1BB, UK.
}

Received: September 28, 1998

Final version: December 17, 1998

\begin{abstract}
Voltammetry does not have the capacity to identify detected compounds unequivocally but relies largely upon independent verification criteria. Nevertheless, the most useful starting points are the size, shape and position of the oxidation peaks. While constant for many compounds, some signals, such as serotonin, change with time and can confound attempts at identification. In the present article we have used voltammogram sequences to generate 'landscapes' as an aid to detection and identification of the constituents of electrochemical signals. This enables one to integrate temporal information into the signal identification criteria and allows the detection of electrochemical features that may be missed on single scans. We report several instances of applications where voltammogram landscapes aid detection.
\end{abstract}

Keywords: Serotonin (5-HT), Dopamine (DA), Fast cyclic voltammetry, Brain slice, Landscape analysis

\section{Introduction}

The inception of in vivo voltammetry, electrochemical measurements in brain tissue, is most often attributed to the pioneering work of Ralph Adams and his group in the late 1960s and early 1970s. Remarkably, those early measurements of ascorbic acid in the rat striatum [1] have since spawned a wide range of different electrochemical methods which cover the entire repertoire of neurotransmitter release events from the correlation of transmitters with animal behavior down to the measurement of individual neurotransmitter vesicle fusions [2].

Voltammetry differs from other neurotransmitter detection methods in the sense that measurements are made in situ and thus, unlike intracerebral dialysis or push-pull perfusion, it does not inherently have the capacity to identify detected compounds [3]. It is necessary to rely upon independent verification of constituent identity. When adequately achieved, the technique is a powerful tool.

This laboratory and others have frequently emphasized the paramount importance of accurate identification of voltammetric signals. In practice, this means electrochemical, anatomical, pharmacological and electrophysiological criteria applied together. Of these, the first and, in many respects, most useful is the voltammetric signal, particularly the size, shape and position of the oxidation peaks. Although never robust enough to be used as sole criterion of identification, it can nonetheless exclude or include putative candidates. Fast cyclic voltammetry (FCV) at carbon fiber microelectrodes has the further identification asset of reduction as well as oxidation peaks [4].

In the past, the principal electrochemical "signature" used in FCV has been the background current-corrected cyclic voltammogram (CV). In this, a voltammetric signal taken before a stimulus is electronically subtracted from one taken during the transmitter release event [5]. The resultant current profile consists solely of the faradaic information of the compound released. This is, in essence, a "snapshot" of the released compound. Sometimes, this is adequate for simple diagnostic purposes, along with the other criteria outlined above. However, when more complex voltammetric signals are observed, single snapshots are insufficient and a "movie" is required.
In the present article we have examined the use of voltammogram "landscapes" as an aid to the identification of the constituents of FCV electrochemical signals - in other words, entire temporal sequences of voltammograms. This enables one to use temporal information as an integral component of the identification criteria and allows the detection of electrochemical features that may be missed on single scans. This is particularly true for those compounds whose voltammetry is complicated by the development of secondary redox couples or adsorption waves (e.g., serotonin: 5-HT) [6].

\section{Materials and Methods}

Fast cyclic voltammetry at carbon fiber microelectrodes [4] was used to measure the release of monoamine in rat and human brain slices evoked by local application of potassium $\left(\mathrm{K}^{+}\right)$, veratridine or by imposition of ischaemia. These signals were compared with those obtained following direct exposure of the microelectrode to monoamine by flow injection analysis (FIA). In all cases, the support electrolyte was an artificial cerebrospinal fluid (ACSF). ACSF was composed of $\mathrm{NaCl}(124 \mathrm{mM}), \mathrm{KCl}$ (2 mM), $\mathrm{KH}_{2} \mathrm{PO}_{4}(1.25 \mathrm{mM}), \mathrm{MgSO}_{4}-7 \mathrm{H}_{2} \mathrm{O}(2 \mathrm{mM}), \mathrm{NaHCO}_{3}$ (25 mM), $\mathrm{CaCl}_{2}(2 \mathrm{mM})$ and $(+)$-glucose $(11 \mathrm{mM})$. The solution was gassed with $95 \% \mathrm{O}_{2} / 5 \% \mathrm{CO}_{2}$ for 1 hour before use.

\subsection{Fast Cyclic Voltammetry}

Figure 1 shows the basic principles of FCV recording. The potentiostat input voltage (Fig. 1A) consisted of $1 \frac{1}{2}$ cycles of a triangular waveform $(-1000$ to $+1400 \mathrm{mV}$ vs. $\mathrm{Ag} / \mathrm{AgCl}$, scan rate: $480 \mathrm{~V} / \mathrm{s}$ ) applied once every four seconds with the working electrode disconnected between scans. The current output of the working electrode (Fig. 1B) was displayed on a Nicolet 310DD digital storage oscilloscope and thence stored onto a floppy disk. Background current signals were subtracted from those in the presence of monoamine to yield the faradaic current resulting from oxidation and subsequent reduction (Fig. 1C). These signals 

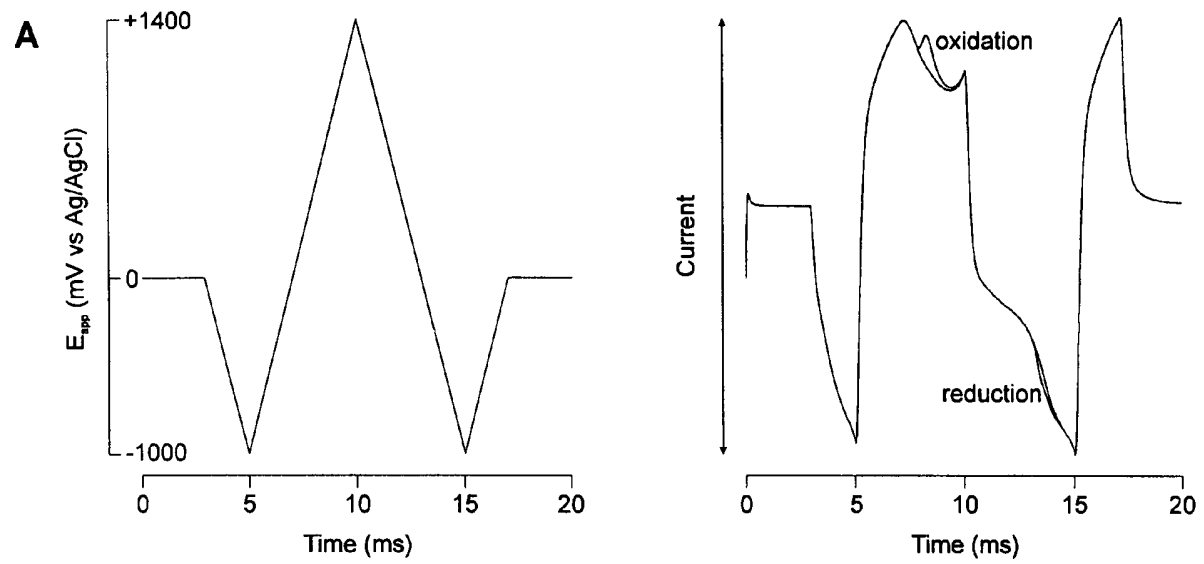

B
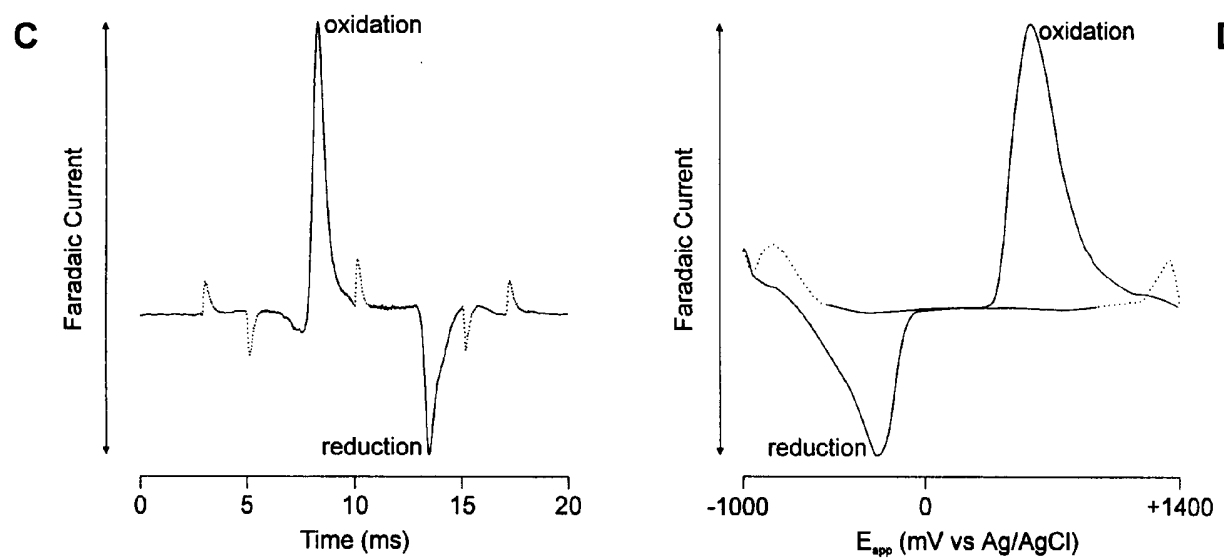

Fig. 1. Waveforms of fast cyclic voltammetry. A) Applied voltage waveform [ $1 \frac{1}{2}$ cycles, -1000 to $+1400 \mathrm{mV}$ (vs. $\mathrm{Ag} / \mathrm{AgCl}$ ), $\left.480 \mathrm{~V} / \mathrm{s}\right]$. B) Current at the working electrode (background and in the presence of dopamine, $5 \mu \mathrm{M}$ ) showing oxidation and reduction regions. C) Faradaic current for the dopamine after subtraction of background current showing oxidation and reduction current peaks. Dotted lines are switching transients. D) Background current-corrected cyclic voltammogram.

could be plotted against the input voltage to yield a conventional background current-corrected cyclic voltammogram (Fig. 1D).

The working electrode consisted of a glass-coated carbon fiber (7 $\mu \mathrm{m}$ diameter) microelectrode [7] cut to $50 \mu \mathrm{min}$ length. The auxiliary electrode was a narrow $(0.5 \mathrm{~mm})$ stainless steel wire and the reference electrode comprised a silver/silver chloride $(\mathrm{Ag} / \mathrm{AgCl})$ cylinder in an ACSF salt bridge.

\subsection{Human and Rat Brain Slices}

Rat brain slices $(350 \mu \mathrm{m}$ thick) were prepared as previously described [8], transferred to a superfusion-type brain slice chamber [9] and held on a stainless steel grid by a nylon mesh frame. The slices were superfused with warmed $\left(32^{\circ} \mathrm{C}\right)$ oxygenated ACSF at $1 \mathrm{~mL} / \mathrm{min}$ throughout the experiment.

Human brain slices were taken from small segments of frontocortical tissue resected for surgical access to deep subcortical tumors. The tissue was collected from the operating theater in ice-cold ACSF and transported to the laboratory two floors above, where it was sectioned $(500 \mu \mathrm{m})$ in the same way as the rat tissue.

The tip of the carbon fiber microelectrode was inserted about $80 \mu \mathrm{m}$ below the surface of the slice. The needle tip of a Hamilton microsyringe was placed approximately $250 \mu \mathrm{m}$ from the working electrode, just above the surface of the slice.
Reference and auxiliary electrodes were positioned at a convenient location in the chamber away from the tissue.

\subsection{Flow Injection Analysis (FIA)}

Flow injection analysis was used for in vitro modeling of monoamine efflux profiles. A simple flow cell with a $2 \mathrm{~mm}$ internal diameter and $60 \mathrm{~mm}$ path was connected to a standard Rheodyne injection valve. The tip of the carbon fiber microelectrode was inserted into the end of the flow cell which was perfused, via a syringe pump, with ACSF at $1 \mathrm{ml} / \mathrm{min}$. A $500 \mu \mathrm{L}$ flow injection loop was then filled with monoamine in ACSF at the desired concentration. At a given time the valve was opened such that the loop contents were inserted into the flow stream past the microelectrode.

\subsection{Stimulation Procedure}

Transmitter efflux was evoked in the brain slices by local application of a small volume of $\mathrm{K}+$ or veratridine. The $\mathrm{K}^{+}$ solution consisted of ACSF with $120 \mathrm{mM}$ of $\mathrm{KCl}$ substituted for $120 \mathrm{mM} \mathrm{NaCl}$ (i.e. the solution contained $122 \mathrm{mM} \mathrm{KCl}$ and $4 \mathrm{mM} \mathrm{NaCl}$ ). Veratridine, dissolved in ACSF, was applied at $100 \mu \mathrm{M}$. All stimulations were applied manually via a Hamilton 
PB-600 dispenser which ejected $20 \mu \mathrm{L}$ of solution from the syringe

\subsection{Cerebral Ischaemia}

Cerebral ischaemia was induced in striatal slices as previously described [10]. Briefly, rat striatal slices were superfused $(400 \mathrm{~mL} / \mathrm{h})$ with ACSF at $34^{\circ} \mathrm{C}$. Episodes of "ischaemia" were mimicked by replacement of $\mathrm{O}_{2}$ with $\mathrm{N}_{2}$ and reduction in glucose concentration from 4 to $2 \mathrm{mM}$. The ensuing DA release was monitored by FCV, as above [4].

\subsection{Data Analysis}

Consecutive waveforms (one "background" and then test waveforms for every four seconds) were initially acquired and stored on floppy disk via a Nicolet 310DD digital storage oscilloscope before being downloaded onto an IBM compatible PC. The background was subtracted from each of the test signals to give a time series of CVs. These were then digitally "filtered" using a series of 3 and 5 point smoothing to eliminate high frequency (ca. $100 \mathrm{kHz}$ ) noise without affecting faradaic information. The signals were then simultaneously plotted using a landscape (waterfall) display. All data handling was performed using "FAMOS” (IMC Mess-Systeme GmbH, Berlin).

\subsection{Drugs and Chemicals}

All chemicals used to make the ACSF were of standard AnalaR grade and obtained from BDH Lab supplies, Poole, UK. Serotonin (5-hydroxytryptamine, 5-HT), dopamine (DA), melatonin and veratridine were purchased from Sigma, Poole UK.

\section{Results and Discussion}

As illustrated in Figure 1, the basic protocol used for the majority of FCV acquires and compares only single faradaic waveforms with reference standards. Whilst for many compounds, this is adequate, it can present problems particularly for those in which the faradaic signal changes during the course of several measurements.

\subsection{Identification of In Vivo Signals}

This concern first came to our attention during studies of 5-HT efflux in brain slices. Even in areas where we knew 5-HT to be the sole compound detected, the faradaic signals sometimes showed only modest similarity to those of reference 5-HT samples. Preliminary investigation showed that this was the result of time-dependent phenomena, whereby the redox signal shape
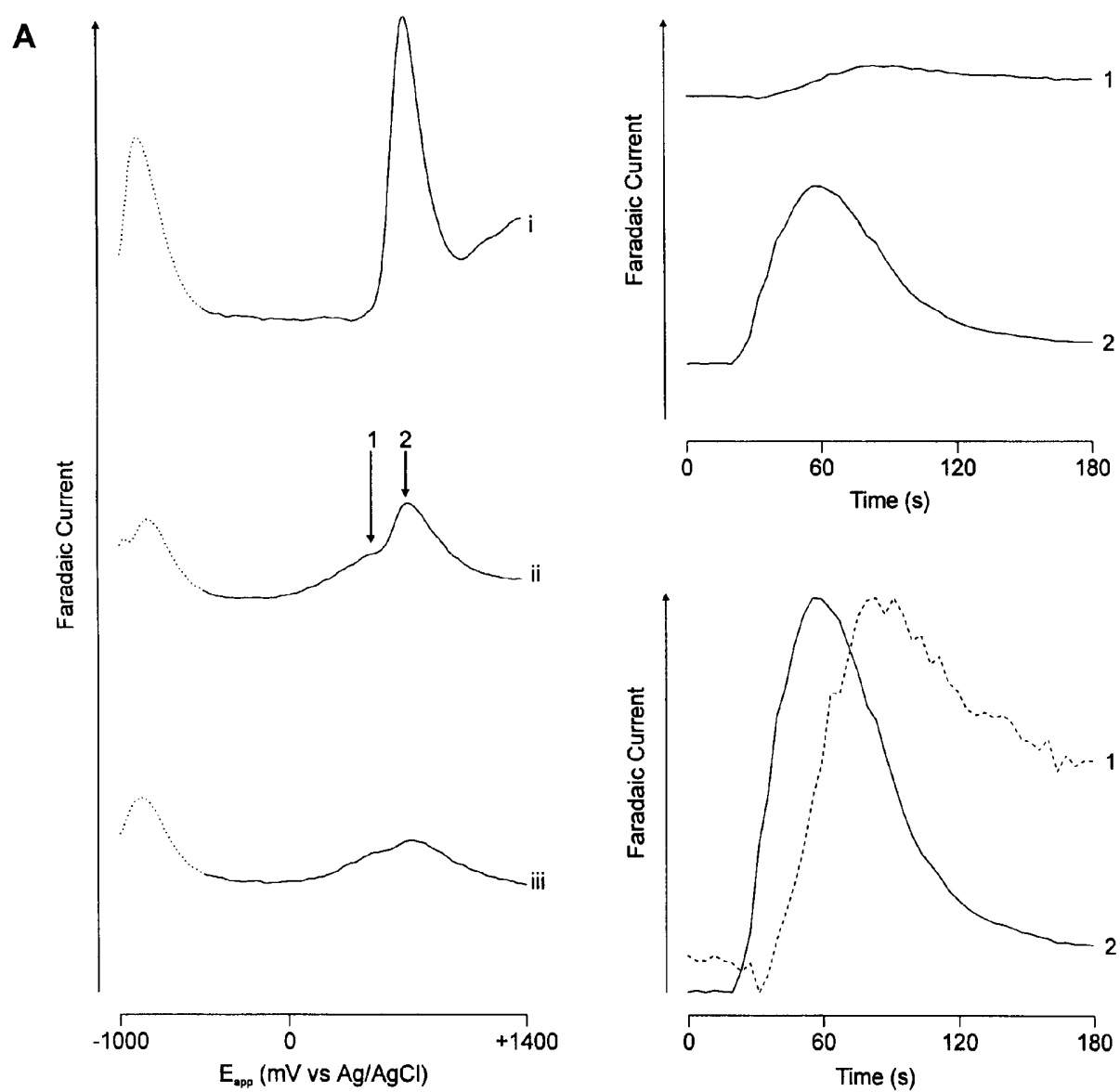

\section{B}

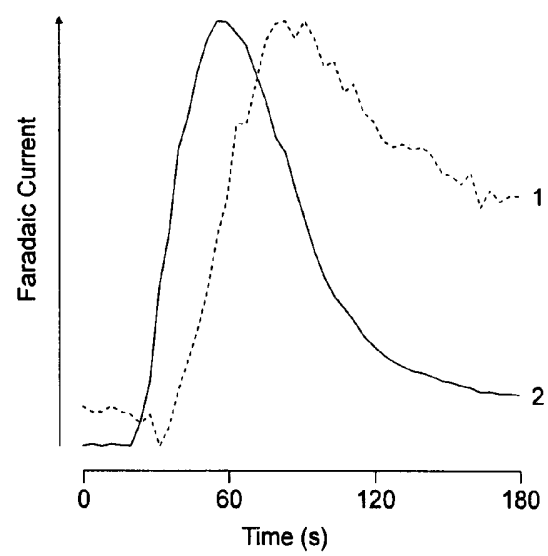

Fig. 2. Changes in faradaic oxidation current signals for 5-HT. A) Faradaic signals for 5-HT $(1 \mu \mathrm{M})$ vs. applied voltage 7, 22 and 37 scans after application of 5-HT to the electrode by flow injection analysis (i, ii and iii, respectively). 1 and 2 are sampling points. B) Time course of changes at sampling points 1 and 2. C) As for B but normalized to the same size, for clarity. 
changed as a function of how long the electrode was exposed to 5-HT.

This is illustrated in Figure 2A. The top signal (i), shortly after addition of 5-HT $(1 \mu \mathrm{M})$, shows a sharp oxidation peak. Signals acquired later (ii and iii), show the development of an early "shoulder" on the oxidation peak. Thus, by comparison of in vivo signals with inappropriate time points in a calibration, it is easy to obtain misleadingly poor fits between endogenous and exogenous 5-HT.

As can be seen, different features of the oxidation of 5-HT have different time courses. If the main oxidation signals (labelled 1 and 2 in Fig. 2A) are sampled and plotted with respect to time (Fig. 2B), it is clear that, in addition to different magnitudes, the responses are also characterized by different time constants. This is most clearly illustrated in Figure $2 \mathrm{C}$, where the signals are normalized for size and polarity. The main oxidation peak (trace 2) shows the sharpest temporal response whilst the "shoulder" (trace 1) is slower to develop and persists longer.

We therefore examined the ways in which the signal for 5-HT changed during a brief application to a carbon fiber microelectrode in vitro. Figure 3 shows "landscapes" of faradaic signals acquired before, during and after the application of 5-HT $(100 \mathrm{nM})$ to the electrode either in vitro (A) or in vivo (C). The signals are shown as pairs, the lower trace of each pair being a digital inversion of the upper.

Thus traces in Figure 3A show the sharp oxidation peak signals characteristic of 5-HT. Also visible is the late oxidation wave and the development of the early oxidation "shoulder" (seen more clearly on the inverted set beneath). The lower traces (Fig. 3C) show the release and removal of monoamine in a slice of rat suprachiasmatic nucleus following local application of veratridine $(20 \mu \mathrm{L}, 100 \mu \mathrm{M})$. Again the features of 5 -HT are readily apparent in the sharp oxidation peak and development of the shoulder during the time course of the recording.

By comparison, DA signals are much simpler. Figure 3B shows "landscapes" of faradaic signals acquired before, during and after the application of DA $(2 \mu \mathrm{M})$ to the electrode in vitro (A). Figure $3 \mathrm{D}$ shows monoamine release by $\mathrm{K}^{+}$depolarization $(20 \mu \mathrm{L}, 120 \mathrm{mM} \mathrm{KCl})$ in rostral-dorsolateral neostriatum. In each case, a single oxidation peak is visible-good evidence that a catecholamine is the sole detected oxidizable species.

\subsection{Study of Complex Electrochemical Reactions}

Landscapes can also be used in vitro to provide qualitative analysis of follow-up reactions. Figure $4 \mathrm{~A}-\mathrm{C}$ shows both oxidation and reduction peak signals for three compounds of increasing redox complexity - DA, 5-HT and melatonin. The redox electrochemistry of DA (Fig. 4A) consists of a single redox couple indicating that the compound oxidized on the anodic scan is reduced to its starting point on the cathodic sweep. The signals for 5-HT (Fig. 4B) show a more complex pattern. The development of secondary peaks from the primary oxidation signal suggests the generation of adsorption prewaves or the induction of follow-up reactions. The electrochemistry of melatonin

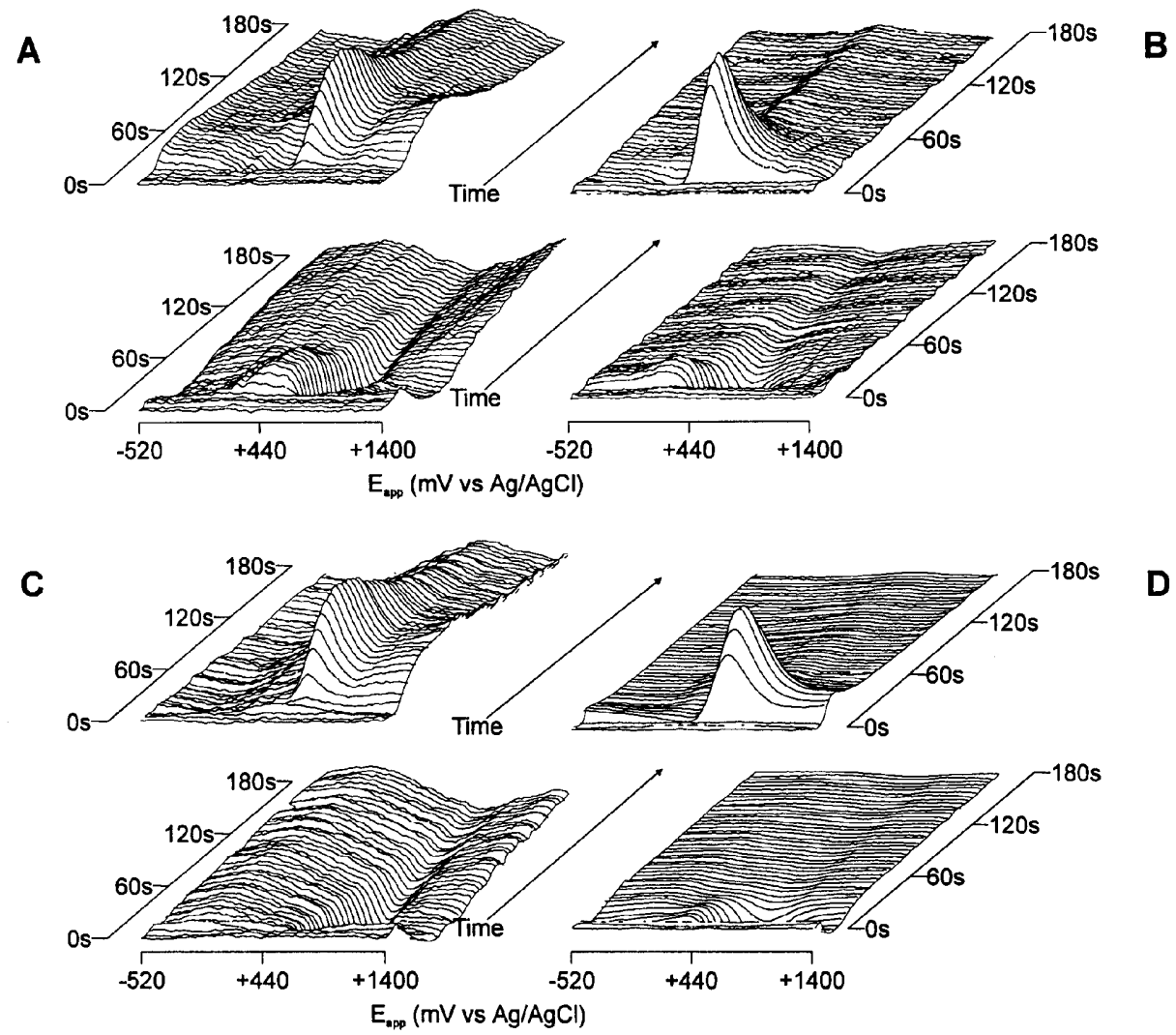

Figure 3. Voltammogram "landscapes" of 5-HT and DA in vitro and in vivo. Sequences of the oxidation $(-520$ to $+1400 \mathrm{mV})$ regions of background current-corrected voltammograms for 100nM 5-HT (A) and $2 \mu \mathrm{M}$ DA (B) applied to the electrode by flow injection analysis. Signals C and D were obtained following local stimulation in the rat suprachiasmatic nucleus $(20 \mu \mathrm{L}$ of veratridine, $100 \mu \mathrm{M})$ or dorsolateral striatum $\left(20 \mu \mathrm{L}\right.$ of $\left.\mathrm{K}^{+}, 120 \mathrm{mM}\right)$, respectively. In each case, the lower panels of each current pair are digital inversions of the upper set. Oxidation peaks are shown upwards in the upper traces and downwards in the lower traces. 
(Fig. 4C) is more complex still with a further two oxidation signals developing from the primary peak. At least one of these is likely (by analogy with methylated catecholamine oxidation) to be the result of $o$-demethylation to form $\mathrm{N}$-acetyl serotonin which then subsequently reacts similarly to $5-\mathrm{HT}$.

\subsection{Faradaic Electrochemistry in Situations of Changing pH}

We have also found this landscape approach useful in circumstances where peaks may shift as a result of $\mathrm{pH}$ changes in the extracellular milieu. Although in an analytical context, this is highly undesirable, such situations may be biologically unavoidable. It is well known that extracellular $\mathrm{pH}$ shifts are asso- ciated with changes in neuronal activity [11]. During pathological events such as cerebral ischaemia there are particularly large (as much as $1 \mathrm{pH}$ unit) reductions in extracellular $\mathrm{pH}$. These precede the rapid transmitter release episodes that can be measured by voltammetry [10].

Figure 5A shows a typical trace in which voltammetric landscapes were recorded in a striatal brain slice during imposition of an hypoxic/hypoglycaemic (ischaemic) insult. Although initially the oxidation current is flat, marked distortion develops in the oxidation signals during the first few minutes of ischaemia, presumably due to the $\mathrm{pH}$ change. Shortly thereafter, there is a sudden transient release of DA, as previously reported [11] Figure 5B shows sample and hold records of current at $+300 \mathrm{mV}$ and $+600 \mathrm{mV}$ vs. $\mathrm{Ag} / \mathrm{AgCl}$ plotted against time. In both, the $\mathrm{pH}$ shift in the background signal is evident immedi-

A

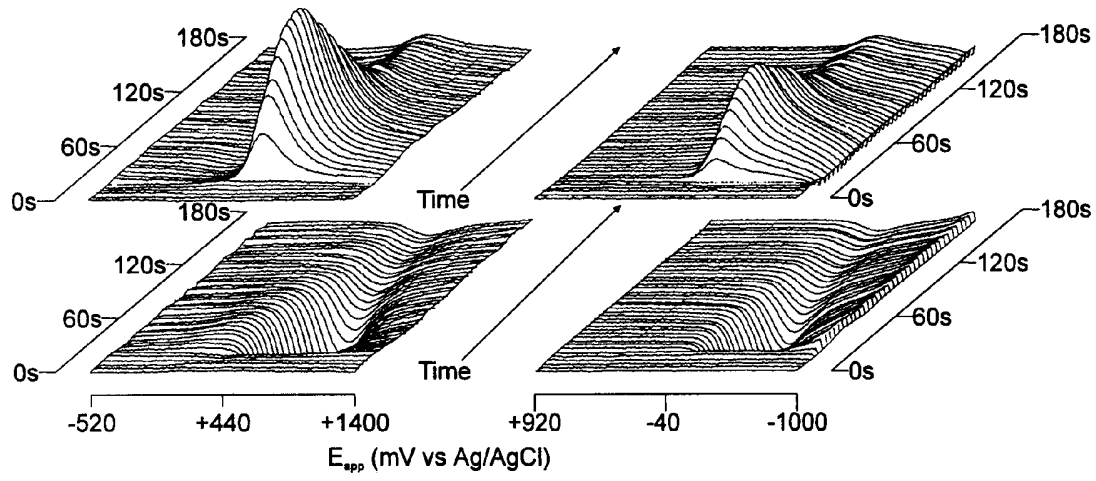

B
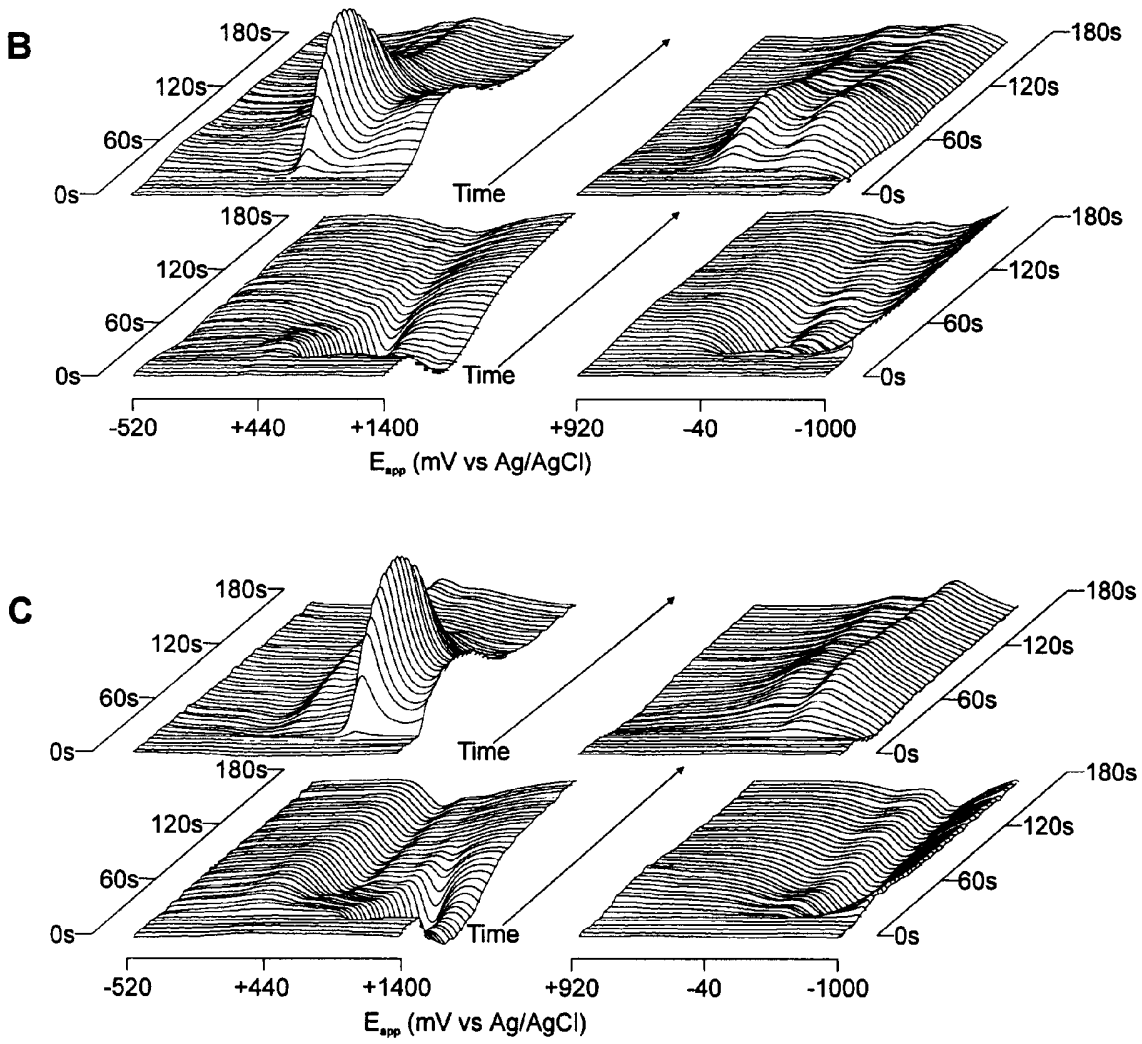

Fig. 4. Sequences of the oxidation $(-520$ to $+1400 \mathrm{mV}$, left panels) and reduction ( +920 to $-1000 \mathrm{mV}$, right panels) regions of background currentcorrected voltammograms for DA $(\mathrm{A}: 10 \mu \mathrm{M}), 5-\mathrm{HT}(\mathrm{B}: 1 \mu \mathrm{M})$ and melatonin $(\mathrm{C}: 50 \mu \mathrm{M})$ applied to the electrode by flow injection analysis. In each case, the lower panels of each pair are digital inversions of the upper set. Oxidation and reduction peaks are shown upwards in the upper traces and downwards in the lower traces. 

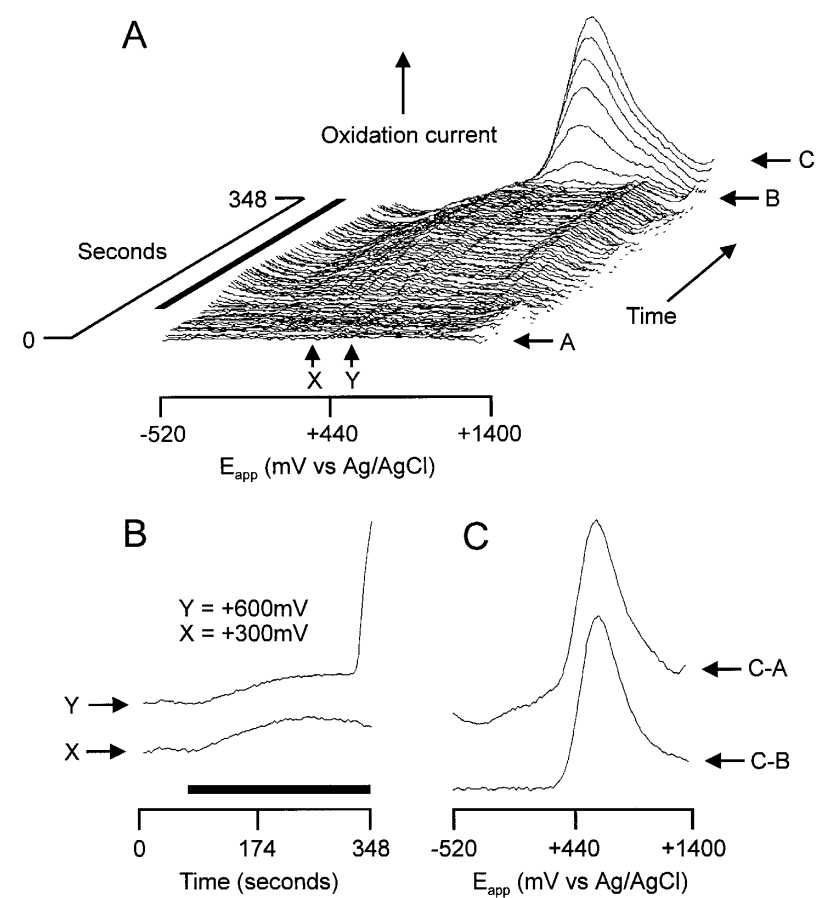

Fig. 5. Dopamine release during cerebral ischaemia. A) Sequences of the oxidation $(-520$ to $+1400 \mathrm{mV}$ regions of background current-corrected voltammograms obtained during ischaemia in a striatal slice. The period of ischaemia is denoted by the solid bar in the $\mathrm{Z}$ axis. B) Sample-andhold records of current at points $\mathrm{X}$ and $\mathrm{Y}(+300$ and $+600 \mathrm{mV}$ vs $\mathrm{Ag} / \mathrm{AgCl}$ ) versus time. C) Oxidation current peaks derived from subtractions of scans at initiation of recording (point A) or initiation of DA release (point B) from peak DA release (point C).

ately after the onset of ischaemia, whilst only in the latter sample point is the DA release apparent.

This $\mathrm{pH}$ shift can easily cause misleading changes in individual voltammograms. Figure 5C shows two background currentcorrected oxidation scans in which the background selected for subtraction differed. In the lower trace, a background current signal immediately prior to DA release (point B) was subtracted from one at peak DA release (point $\mathrm{C}$ ). In this case, the DA oxidation peak is clean and monophasic. However, if an inappropriate background signal, such as at the beginning of recording (point $\mathrm{A}$ ), is chosen, the resultant oxidation profile shows an apparent shoulder before the oxidation peak, suggestive of 5-HT. Such an interpretation is, of course, wholly erroneous and due to the incorporation of a $\mathrm{pH}$ shift into the data. This, in particular, emphasizes the value of landscape presentation (Fig. $5 \mathrm{~A}$ ) where such shifts are obvious and may be more readily excluded from the data.

\subsection{Signals Close to the Detection Limit}

In cerebral ischaemia, the neurotransmitter dynamics are such that, although distorted by $\mathrm{pH}$ shifts, peaks may still easily be detected. However, this is not always the case. In circumstances where the quality of the tissue cannot always be ensured, $\mathrm{pH}$ shifts may dominate the voltammograms to the extent that faradaic information is overwhelmed. This is often the case with human brain tissue which, almost by definition, is abnormal (few neurosurgeons would knowingly operate on healthy brain!).
Figure 6 shows a recording of veratridine-stimulated transmitter release in a human frontal cortex brain slice. Although the dominant feature of the landscape (Fig. 6A) is the $\mathrm{pH}$ change induced by the increased sodium influx (arrow X), there is clear evidence of transmitter release with an apparent peak at point $\mathrm{Y}$. Although the signals are too close to the noise to be identified, the landscapes nevertheless enable the detection of features that are not evident on individual scans taken from the series (Fig. 6B).

\section{Conclusions}

This improvement to the FCV methodology may ultimately provide a basis by which mixtures of compounds may ultimately be resolved. This is a subject of considerable interest in FCV and has been addressed in other electrochemical [12] and mathematical ways [13]. Although unlikely that FCV can approach the resolution of the slower scanning methods, it is apparent that some enhanced resolution may be attainable and that it may be possible to measure catecholamines and indoleamines together in areas with complex innervation, such as the thalamus or to detect faradaic information in circumstances where charging current changes occur.

Irrespective of the above, the use of sequences (landscapes) of faradaic signals allows redox features to be distinguished that are less readily apparent on single traces. To some degree this hinges upon the high capacity of the human eye to detect detail close to the noise level of the system. However, the confirmation by multiple sample-and-hold analysis shows that this is not subjective and suggests that this may be a worthwhile additional tool for signal identification. With practice, low level signals may be extracted from noise. Further improvements in sensitivity and

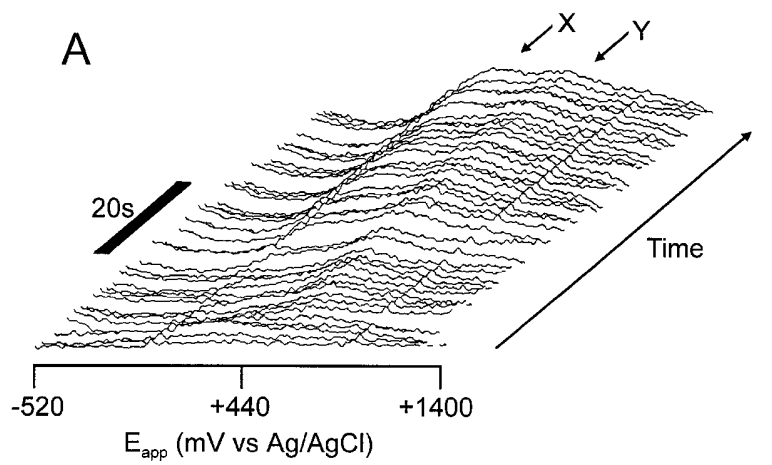

B

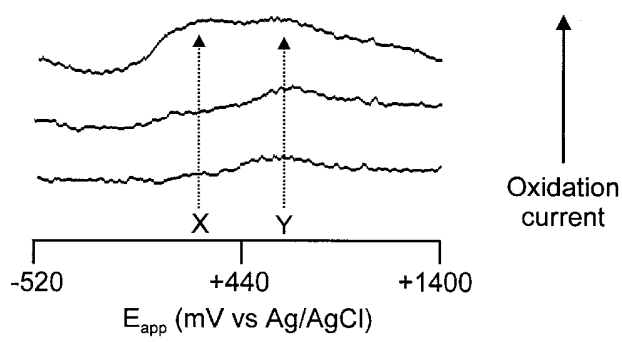

Figure 6. A) Landscape of consecutive anodic CVs taken during a veratridine stimulation $(20 \mu \mathrm{L}, 0.1 \mathrm{mM})$ in a slice of human frontal cortex. Note the early "peak" (X) has a slower rise time than the later peak (Y). B) Three randomly chosen individual oxidation scans illustrating the difficulty in obtaining clear faradaic information (at point $\mathrm{Y}$ ) from signals close to the noise level and in which $\mathrm{pH}$ shifts (point $\mathrm{X}$ ) occur. 
data acquisition times are being sought to allow the method to be applied to smaller and more rapid signals.

\section{Acknowledgements}

This research was funded, in part, by the Wellcome Trust (grant no 038937/1.5). We thank Dr Christopher Toner for the data upon which Figure 5 is based.

\section{References}

[1] P.T. Kissinger, J.B. Hart, R.N. Adams, Brain Res. 1973, 55, 209.

[2] Voltammetric Methods in Brain Systems (Eds: A.A. Boulton, G.B. Baker, R.N. Adams), Humana Press, Totawa.
[3] C.A. Marsden, M.H. Joseph, Z.L. Kruk, N.T. Maidment, R.D. O’Neill, J.O. Schenk \& J.A. Stamford, Neuroscience 1988, 25, 389.

[4] J.A. Stamford, J. Neurosci. Meth. 1990, 34, 67.

[5] J. Millar, J.A. Stamford, Z.L. Kruk, R.M. Wightman, Eur. J. Pharmacol. 1985, 109, 341.

[6] J.J. O'Connor, Z.L. Kruk, J. Neurosci. Meth. 1991, 38, 25

[7] M. Armstrong James, J. Millar, J. Neurosci. Meth 1979, 1, 279.

[8] P. Palij, J.A. Stamford, Brain Res. 1992, 587, 137.

[9] C.D. Richards, W.J.B. Tegg, Br. J. Pharmacol. 1977, 59, 526P.

[10] C.C. Toner, J.A. Stamford, J. Neurosci. Meth. 1996, 67, 133

[11] E. Sykova, A. Chvatal, J. Chem. Neuroanat. 1993, 6, 247.

[12] J.A. Stamford, Z.L. Kruk, J. Millar, Brain Res. 1990, 515, 173.

[13] J.L. Gonzalez-Mora, Z.L. Kruk, in Monitoring Molecules in Neuroscience (Eds: A. Louilot, T. Durkin, U. Spampinato, M. Cador.), CNRS, Univ. Bordeaux 1994, p. 3. 